\title{
A descriptive and model-based spatial comparison of the standardised mortality ratio and the age-standardised mor- tality rate
}

\author{
Sibylle Sturtz, Katja Ickstadt \\ Department of Statistics, University of Dortmund, Dortmund, Germany
}

\begin{abstract}
The number of deaths in a particular connection can be expressed in different ways. In spatial epidemiology, two widely used measures are the standardised mortality ratio (SMR) and the so called mortality rate. This paper compares these two ways of expressing mortality using a descriptive and a model-based approach. Age-standardised versions of both terms have been investigated by a descriptive analysis of temporal and spatial patterns and by employing different Bayesian spatial models to study their performance. We observed that the SMR and the age-standardised mortality rate (ASM) are strongly correlated and lead to comparable results. This demonstration is based on mortality data by age, stratified into five-year ranges, from the cause-of-death-statistics with reference to ischaemic heart disease and lung cancer in 54 counties of the German state of North Rhine Westphalia between 1980 and 1997.
\end{abstract}

Keywords: standardised mortality ratio, mortality rate, ischaemic heart disease, lung cancer, statistics, Bayesian spatial models.

\section{Introduction}

In the analysis of mortality at the unit of administrative districts, raw counts alone are not helpful: the data must be standardised and the population at risk taken into account. If data for different agegroups are available, these should also be taken into account. Two different expressions are widely used, i.e. the standardised mortality ratio (SMR) which is arrived at by dividing the number of observed cases by those expected, a figure related to indirect standardisation, while the mortality rate is the number of deaths per 100,000 persons, a figure related to direct standardisation. For both, age-standardised versions can be calculated leading to the age-standardised SMR (denoted by SMR*) and the age-standardised mortality rate (ASM). Assuming a homo-

Corresponding author:

Sibylle Sturtz

Department of Statistics, University of Dortmund

44221 Dortmund, Germany

Tel. +49 231755 4391; Fax +49 2317555303

E-mail: sturtz@statistik.uni-dortmund.de geneous risk of disease across all persons at risk, the expected number of cases can be calculated. The main goal of our paper is to compare SMR*s and ASMs from a descriptive angle as well as from a model-based point of view. It should be noted that we do not compare the SMRs with the comparative mortality figures (CMFs) which would be the corresponding figures based on direct standardisation, since these are not as widely used.

The cardiovascular mortality in British towns has been analysed by Pocock et al. (1982) using SMRs and a similar data set was studied by Gardner (1973) using mortality rates per 100,000 persons. Other spatial examples based on SMRs have been given by Clayton and Kaldor (1987), Mollié and Richardson (1991), Mollié (1996) and Knorr-Held and Becker (2000). Spatial analyses based on mortality rates have been presented by Tsutakawa et al. (1985), Pickle et al. (1999) and Mungiole et al. (1999). Further, Knorr-Held and Besag (1998) have put forward a spatial-temporal analysis. These are sensible approaches for the analysis of mortality figures in time and space but one has to decide 
whether to use SMRs or ASMs. In disease mapping, the common approach is to use indirectly standardised SMRs (Clayton and Kaldor, 1987) which assumes the multiplicative model to be valid (Breslow and Day, 1975). Nevertheless, a comparison of two mortality measures is difficult when the underlying study populations are not the same. The assumption of uniformity across different agegroups may lead to fallacy (Rothman and Greenland, 1998). While Breslow and Day (1975) show that it is sufficient if the area and age effects are independent with respect to the standard population, Pickle and White (1995) maintain that the resulting differences are negligible as long as the rank order of the area is preserved. Freeman and Holford (1980) show that comparison of both, directly and indirectly adjusted rates on a linear or a ratio scale, is valid under the multiplicative model. For indirectly standardised rates the standard population is required to belong to the same class of multiplicative models.

The present work is an investigation into the performance of the two common expressions of mortality, i.e. the mortality ratio and the mortality rate. Using a descriptive analysis of temporal and spatial patterns, as well as employing different spatial models in a Bayesian framework, always assuming a multiplicative model for the data, we find that both terms are comparable and lead to similar results. After defining both terms in detail, it can be seen that they differ numerically from each other by a multiplicative factor in the analysis of just one age group. This does not hold for age-standardised data. In the present paper, we compare the spatial and temporal performance of SMR*s and ASMs descriptively, and then we demonstrate the performance of spatially-modelled data.

\section{Defining the mortality terms}

In this section we first present the definitions and go on to discuss some of the features of the measures used (SMR and ASM). For an introduction on standardization of rates and ratios Fleiss et al. (2003) may be consulted. Regarding the need of standardisation, Waller and Gotway (2004) have produced an illustrative example.

\section{The standardised mortality ratio (SMR)}

The SMR is defined as the ratio of the observed deaths and those expected. In comparing mortality measures, for example for an infectious disease in two different areas, the number of expected deaths can naturally be inferred as that of a group of unexposed people. If the mortality measures of a comparable population in time and space are not available, the number of expected deaths $\left(E_{i, t}\right)$ in each administrative district $(i), i=1, \ldots, n$, in the year $(t), t=1, \ldots, T$, can be calculated as the average disease rate $(\lambda)$ for the whole study region multiplied by the population at risk, i.e.

$$
E_{i, t}=\lambda * P_{i, t}=\frac{\sum_{i=1}^{n} \sum_{t=1}^{T} O_{i, t}}{\sum_{i=1}^{n} \sum_{t=1}^{T} P_{i, t}} * P_{i, t},
$$

where $O_{i, t}$ denotes the number of observed deaths in region $i$ during the year $t . P_{i, t}$ is the study population. The SMR in the area under study (i) during a particular year $(t)$, denoted by $\mathrm{SMR}_{i, t}$, is then equal to $\mathrm{O}_{i, t}$ divided by $\mathrm{E}_{i, t}$, which can be expressed mathematically by the following equation:

$$
\mathrm{SMR}_{i, t}=\frac{O_{i, t}}{E_{i, t}} .
$$

When information about different age-groups is available, the SMR* can be calculated, using the following formula:

$$
\mathrm{SMR}^{*}{ }_{i, t}=\frac{\sum_{k=1}^{K} O_{i, t, k}}{\sum_{k=1}^{K} E_{i, t, k}}=\frac{\sum_{k=1}^{K} O_{i, t, k}}{\sum_{k=1}^{K} \lambda_{k} P_{i, t, k}}
$$


where the index $k=1, \ldots, K$ denotes the age group and the average disease rate $\lambda_{\mathrm{k}}$ for each age group is calculated as:

$$
\lambda_{k}=\frac{\sum_{i=1}^{n} \sum_{t=1}^{T} O_{i, t, k}}{\sum_{i=1}^{n} \sum_{t=1}^{T} P_{i, t}} .
$$

Both $\mathrm{SMR}_{i, t}$ and $\mathrm{SMR}^{*}{ }_{i, t}$ are standardised indirectly, as we estimated the number of expected cases in a given population by rates and age-specific rates, respectively, according to, e.g., Waller and Gotway (2004). Usually, $O_{i, t}\left(O_{i, t, k}\right)$ is taken to follow a Poisson distribution conditional on $E_{i, t}\left(E_{i, t, k}\right)$. In spatial analysis, these rates $\left(\lambda_{k}\right)$ have the advantage of being equal to 1 if the expected number of deaths is equal to the observed one. In a spatio-temporal analysis, the expected values of the SMR *s are timedependent and the observations for each year can no longer be taken to follow a Poisson distribution with the parameter $\lambda_{k}=1$. Instead, a Poisson process with time-varying $\left(\lambda_{k}\right)$ must be assumed.

\section{The age-standardised mortality rate (ASM)}

As an alternative to SMR one can employ ASMs modelled by normal distribution. However, for rare causes-of-death this model only holds after a suitable transformation, e.g., after a log-transformation of the ASMs. When, on the other hand, information on different age-groups is available, it is possible to standardise mortality rates, either directly or indirectly. Pickle and White (1995) have put forward the conditions, under which directly and indirectly standardised rates produce numerically identical results. In this paper, instead of choosing indirect standardisation as for the SMRs, we concentrated on direct standardisation for rates which is more frequently used. For this approach, however, a standard population is required and a useful choice is to employ Segi's world population as discussed by Becker et al. (1984).

The weighted sum of the standardised mortality rates in the region $(i), i=1, \ldots, n$, in the year $(t)$, $t=1, \ldots, T$, is then defined by

$$
\mathrm{ASM}_{i, t}=\sum_{k=1}^{K} g_{k} \frac{O_{i, t, k}}{P_{i, t, k}} * 100,000
$$

where $g_{k}, k=1, \ldots, K$, are weights given by the proportions of a standard population.

In a purely spatial analysis, the interpretation of ASMs is more difficult than that of SMR"s since an expected value for use as reference is usually not available. In temporal analyses, the use of ASMs has the advantage that information about the number of deaths in each temporal unit is still available whereas, for SMRs, it is not. Since normally distributed (possibly transformed) rates with varying expected values for different years can be assumed, a Gaussian process is a suitable model.

\section{Relation of the mortality ratio and the mortality rate in one age group}

Applying the equations nos. 1 and 2 above to one age group $(K=1)$ exclusively, results in a constant ratio between the overall ASM and SMR for all regions $i$ and all years $t$ together, i.e.

$$
\frac{\mathrm{ASM}}{\mathrm{SMR}}=\frac{\sum_{t=1}^{T} \sum_{i=1}^{n} O_{i, t}}{\sum_{t=1}^{T} \sum_{i=1}^{n} P_{i, t}} * 100,000=\lambda * 100,000
$$

However, this only holds for the data of one agegroup. The relation for age-standardised numbers is discussed below. Furthermore, it should be remembered that equation no. 3 is only applicable for the numbers, not for the distribution-theoretic background of the figures. 


\section{The mortality data set}

The data of this study is based on emanates from the state authority dealing with cause-of-death-statistics in the German state of North Rhine Westphalia. The number of deaths and their causes were collected for each of the 54 counties, separately for each sex, from each year between 1980 and 1997. These data were added up in ranges of age groups, each covering 5 years, except the data for the very young who were divided into two groups: those below 1 year of age, and 1 to 14 year-old children. Data regarding persons older than 85 years were collected into one group, while data regarding people whose age was unknown at the time of death, were ignored. The causes-of-death were coded according to the $9^{\text {th }}$ revision of the International Classification of Diseases (ICD9) (DIMDI, 1993). In addition, the corresponding population was also stratified by age group, sex, county and year. Thus, the mortality rates (equation no. 2) and the mortality ratios (equation no. 1) could be calculated as described in the previous section using Segi's world population as the standard population. The underlying population counts were about 9 million for both men and women.

Ischemic heart disease (ICD 410-414), the most frequent cause of death in the group of heart conditions for both sex (Sturtz, 2002) and lung cancer (ICD 162), were considered more closely. These causes-of-death were chosen for the study since they represent different risk patterns. Different mortality numbers in men and women complete the list of different scenarios that can possibly lead to different results in SMR and ASM.

\section{Descriptive comparison of SMR and ASM}

For any selected disease, it is possible to compare the temporal development of both mortality measures under study, e.g., using so called box-plots. Fig. 1 shows examples of these for both SMR and ASM, and also for each year with regard to male deaths due
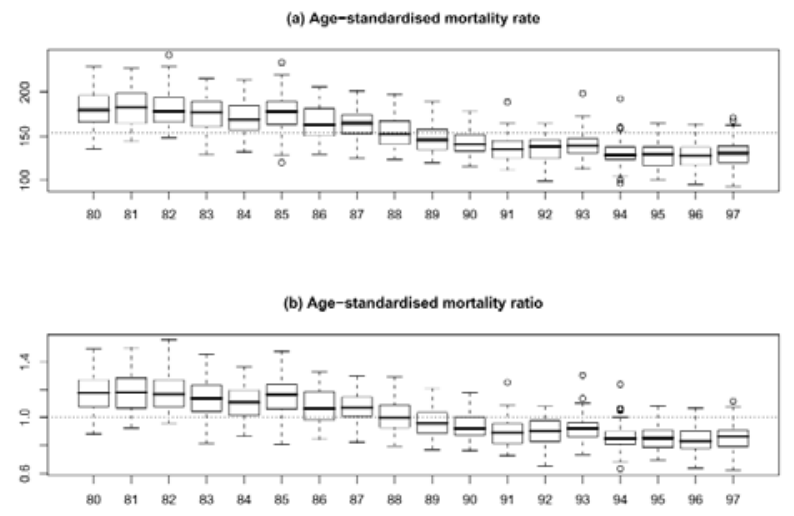

Fig. 1. Temporal development of ischaemic heart disease in men in North Rhine Westphalia, Germany, (a) ASMs, (b) SMR*s.

to ischaemic heart disease. To facilitate comparison, horizontal lines were drawn at $\lambda=1$ for the SMR*s, i.e. the expected value of the mean of the Poisson process for data available for just one year, and for the ASMs at the mean of the rates for all years.

Obviously, the overall numerical trends in the mortality rate and in the mortality ratio are approximately the same. Both exhibit a falling tendency. Even the pattern of outliers is very similar for both as, for example, seen in the year 1994. The mean of the mortality ratio was 1.010 and the variance 0.029 leading to a variation coefficient of 5.955. Naturally, the mortality rates values were higher with a mean of 154.59 and a variance of 725.83 . However, the variation coefficient at 5.738 was approximately the same. The described tendency is reflected in Table 1 . For the three years chosen as example, both the SMR*s and the ASMs decreased with reference to the number of deaths from ischaemic heart disease for men. Although fewer women died from ischaemic heart disease, this tendency holds also for the women. The calculated means of the SMR*s and the ASMs are given in Table 1. To improve the visual comparison, we calculated the Spearman rank correlation (Spearman, 1904), together with the number of counties which had changed quintiles each year. In this analysis, we also included other diseases that show different temporal patterns compared to ischaemic heart disease. These show increasing numbers of deaths over time, as for lung cancer in women, or a fairly sta- 
Table 1. Overall SMR*s and ASMs and rank correlation coefficients for chosen diseases and selected years.

\begin{tabular}{|c|c|c|c|c|c|c|}
\hline Cause-of-death & Sex & Year & SMR* & ASM & $\begin{array}{c}\text { Rank } \\
\text { correlation }\end{array}$ & $\begin{array}{c}\text { Counties } \\
\text { changing quintiles }\end{array}$ \\
\hline \multirow[t]{6}{*}{ Ischaemic heart } & Male & 1980 & 1.186 & 183.07 & 0.986 & 12 \\
\hline & & 1988 & 1.019 & 155.49 & 0.983 & 8 \\
\hline & & 1997 & 0.862 & 131.08 & 0.987 & 14 \\
\hline & Female & 1980 & 1.066 & 72.21 & 0.961 & 21 \\
\hline & & 1988 & 1.021 & 67.23 & 0.948 & 11 \\
\hline & & 1997 & 0.990 & 62.20 & 0.942 & 19 \\
\hline \multirow[t]{6}{*}{ Lung cancer } & Male & 1980 & 1.050 & 59.80 & 0.982 & 8 \\
\hline & & 1988 & 1.050 & 60.33 & 0.989 & 6 \\
\hline & & 1997 & 0.989 & 52.40 & 0.995 & 6 \\
\hline & Female & 1980 & 0.677 & 6.28 & 0.852 & 19 \\
\hline & & 1988 & 8.245 & 8.25 & 0.947 & 12 \\
\hline & & 1997 & 1.241 & 11.76 & 0.906 & 21 \\
\hline
\end{tabular}

ble tendency which holds for lung cancer in men.

For all diseases, the calculated rank correlation coefficient is close to 1 , reflecting a high correlation of both mortality measures. This holds for both diseases and both sex in all years regardless of the different temporal tendency.

When spatial patterns are analysed, the data are normally plotted using some arbitrary classification, e.g., quintiles. To explore the effect of different standardisations, we calculated the number of counties which were assigned to different quintiles in each particular year. The number of change of quintile allocation ranged between $11 \%(6 / 54)$ and $39 \%(21 / 54)$. The deviation was found to be higher for women for both diseases but, for all groups, the portion of quintile changes was of the same magnitude. In our analysis, the proportion of quintile changes came out higher than that reported by Pickle and White (1995) in comparing directly and indirectly standardised rates. However, as these authors used mortality data in the United States with an underlying overall population of around 280 million in 1970-1974, compared to the male population of 9 million in North Rhine Westphalia, their work naturally produced more stable quintile allocations. The rank correlation between direct and indirect standardisation, on the other hand, was high in both our example and in theirs. In summary, the temporal development of both the SMR*s and the ASMs turned out to be generally the same with only minor differences. These occur mainly in assigned percentiles, not in county ranks, and are therefore mainly a problem of interpretation when comparing spatial structures of different years using surface plots.

\section{Model-based comparison of SMR and ASM}

With the aim of investigating if the spatial patterns of the age-standardised numbers varied or behaved in a similar way for different diseases, we plotted the SMR*s for all years given per county against the ASMs (Fig. 2) for ischaemic heart disease and lung cancer.

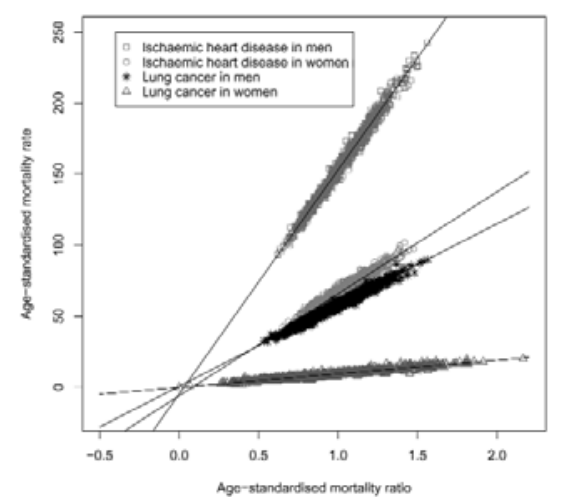

Fig. 2. SMR*s versus ASMs for various diseases for all years given per county and fitted straight lines. 
Having done this, we observed a linear relation with differing slopes and intercepts. The population considered in the study did not seem to have any influence at all; neither on the slope, nor on the intercept as seen when comparing the curves of ischaemic heart disease in women and lung cancer in men. Both have similar slopes, whereas the underlying population was different and vice versa. For the intercept we observed different values in comparing the results for one sex (e.g. lung cancer in women and ischaemic heart disease in women) and therefore the same population. Slopes and intercepts of the fitted lines, calculated by the least squares method, are detailed in Table 2.

The influence of the absolute numbers of deaths is a point worth mentioning. An increasing number of deaths also augments both the slope of the adjusted line and the standard error of the residuals (Table 2). The higher the probability to die due to one of the diseases chosen for study (or any other disease for that matter), the higher the ASM. However, this is only to be expected since it is an estimate of mortality probability. Therefore the slope of the least squares fit increases. Moreover, the coefficient of determination $\mathrm{R}^{2}$, given in Table 2 , shows that, based on the mortality ratio, the mortality rate can be calculated quite precisely.

Knowing that high mortality rates lead to high mortality ratios, the spatial structure of both should be similar. We first performed a descriptive analysis of the observed SMR*s and ASMs for ischaemic heart disease in men for three different years choosing the first and last year (1980 and 1997) of the observation period plus one year in the middle (1988). The range of these data differed widely, which was especially obvious for the SMR*s. The data from 1980, produced a range from 0.881 to 1.495 with a mean of 1.186 , whereas the range was 0.620 to 1.119 in 1997 , with a mean of 0.862 . Secondly, we employed spatial models. Only the results based on the data for ischaemic heart disease were included since the lung cancer data lead to similar results. Three different Bayesian models were utilised. For the ASMs, assumed to either follow a normal distribution directly or to follow a log-normal distribution, it is common to employ an auto-Gaussian model based on Markov Random Fields (Besag, 1974), in our case:

$$
\log \left(\mathrm{ASM}_{i}\right) \sim \operatorname{Gau}\left(\mu_{i} \sigma^{2}\right) \quad \text { where } \mu_{i}=\gamma_{0}+b_{i}
$$

The mean of a Gaussian distribution $\left(\mu_{i}\right)$ is equal to an overall level $\left(\gamma_{0}\right)$ and an individual spatial random effects $\left(b_{i}\right)$. We chose the dispersion parameters for $b_{i}, i=1, \ldots, n$, depending on the variance $\sigma^{2}$ of the Gaussian distribution to ensure identifiably. Otherwise we would have had to estimate two variance terms based on only one observation per area. With this choice, the dispersion parameters $\sigma^{2}{ }_{i}=\sigma^{2} / \mathrm{n}_{i}$ increase with decreasing numbers of the neighbours $\left(n_{i}\right)$ of the region $(i)$. Non-informative prior distributions of $b_{i}, \gamma_{0}$ and $\sigma^{2}$ were chosen as follows:

$$
\begin{aligned}
& b_{i} \sim \operatorname{Gau}\left(\bar{b}_{i} \sigma_{1}^{2}\right) ; \\
& \gamma_{0} \sim \mathrm{U}(-\infty, \infty) ; \text { and } \\
& \sigma^{2} \sim \operatorname{InvGamma}\left(r^{*}, d^{\prime \prime}\right) .
\end{aligned}
$$

\begin{tabular}{|c|c|c|c|c|c|}
\hline Cause-of-death & Sex & Intercept & Slope & $\mathrm{R}^{2}$ & $\begin{array}{c}\text { Standard error } \\
\text { of residuals }\end{array}$ \\
\hline Ischaemic heart & Male & -4.829 & 157.78 & 0.987 & 3.033 \\
\hline Ischaemic heart & Female & -6.071 & 71.79 & 0.931 & 2.704 \\
\hline Lung cancer & Male & 0.574 & 57.24 & 0.977 & 1.531 \\
\hline Lung cancer & Female & -0.162 & 9.57 & 0.923 & 0.934 \\
\hline
\end{tabular}

Table 2. Results of the least squares estimation of various diseases. 
Given its neighbouring sites, as described in Besag et al. (1991), the construction of the mean of the Gaussian distribution for the spatial effects $\bar{b}_{i}$ was based on an intrinsic Gaussian conditional autoregressive model. In addition, the $b_{i}$ values are constrained in summing up to zero (Besag and Kooperberg, 1995). However, this requires an improper and unbounded uniform distribution on the real line for $\gamma_{0}$. The parameters of the Inverse Gamma distribution for the variance parameter $\sigma^{2}$ were chosen as $r^{*}=0.001$ and $\mathrm{d}^{*}=0.1$ implying a mean of 0.01 and a variance of 0.1 . A range of other prior values was tried and led to similar results.

For the SMRs, we assumed the observed cases $\left(O_{i}\right)$ to follow a Poisson distribution with a mean parameter $\left(\mu_{i}\right)$. The $\mu_{i}$ values are dependent on the expected number of deaths $\left(E_{i}\right)$, an overall level $\left(\gamma_{0}\right)$ and individual spatial random effects $\left(b_{i}\right)$ for each county, $i, i=1, \ldots, n$, as expressed by the formula below:

$$
\log \left(\mu_{i}\right)=\log \left(E_{i}\right)+\gamma_{0}+b_{i}
$$

Again, we chose non-informative prior distributions for the parameters

$$
\begin{aligned}
& b_{i} \sim \operatorname{Gau}\left(\bar{b}_{i} \sigma_{1}^{2}\right) ; \\
& \gamma_{0} \sim \mathrm{U}(-\infty, \infty) ; \text { and } \\
& \sigma^{2} \sim \operatorname{InvGamma}\left(r^{*}, d^{*}\right)
\end{aligned}
$$

with $\sigma^{2}{ }_{i}=\sigma^{2} / \mathrm{n}_{i}, r^{*}=0.001$ and $d^{*}=0.1$, similarly to the auto-Gaussian model.

Following Clayton and Kaldor (1987), and also discussed by Besag et al. (1991), the $b_{i}$ values were based on an intrinsic Gaussian conditional autoregressive model. We refer to this model as the Poisson-log-Normal model with spatial random effects. The SMRs of interest for this study were calculated by the following formula:

$$
\mathrm{SMR}_{i}=\frac{\mu_{i}}{E_{i}}
$$

A Markov chain Monte Carlo (MCMC) scheme for calculation of the posterior distribution was set up using the software WinBUGS (Spiegelhalter et al., 2004). For both models, a "burn-in" period of 5,000 iterations was chosen, followed by a sample of 10,000 .

There exist several alternative approaches for modelling Poisson or Gaussian spatial distributed data. Best et al. (2000), for example, modelled the observed counts, which the SMRs are based on, using a Poisson-Gamma model, while Wakefield et al. (2000) used a Poisson-Gamma and a Poissonlog-Normal model. Different approaches, given by Fernandez and Green (2002) and Green and Richardson (2002), have introduced a mixture model for Poisson-distributed data in which the weights vary according to the observations. Another well known approach is the one introduced by Knorr-Held and Raßer (2000). Many of these mortality ratio models using Poisson distributions are clustering-based. For Gaussian distributed data, Cressie (1993) used a spatial autoregressive regression model.

As an alternative of the Poisson-log-Normal model with spatial random effects we employed one of the cluster-based methods, the so called Bayesian Detection of Clusters and Discontinuities in diseases maps (BDCD) model, described by Knorr-Held and Raßer (2000). Although the main goal is clustering, such models are often used as a smoothing tool in disease mapping (Best et al., 2005). As the BDCD model assumes Poisson-distributed data, such as the Poisson-log-Normal model, it only can be used for the counts leading to SMRs. Given the number of observed cases $\left(\mathrm{O}_{i}\right)$ in the region $(i)$, and the number of expected cases $\left(\mathrm{E}_{i}\right)$, we assumed a constant relative risk $\left(h_{c}\right)$ in one or more regions leading to

$$
\mathrm{O}_{i} \sim \operatorname{Pois}\left(E_{1} h_{c}\right)
$$

Regions are treated by grouping them to a cluster $\left(C_{c}\right)$ with associated risk $\left(h_{c}\right)$ which is a partition of the set of all regions. Cluster configuration is changed permanently, for example, by using a birth 
move utilising the reversible jump MCMC method by Green (1995). Alternative moves are, for example, a death move or one shifting the cluster centre to another region. Cluster affiliation and according risks are monitored during the MCMC run and used to calculate Monte Carlo estimates for each subregion. For each cluster, the posterior distribution of relative risk is calculated. The number of clusters, $c$, $c=1, \ldots, n$, as well as the relative risk $\left(h_{c}\right)$ are parameters which have to be specified by a prior distribution. We chose to work with:

$$
\begin{aligned}
& c \sim \mathrm{U}(1, n) ; \\
& \log \left(\mathrm{h}_{\mathcal{c}}\right) \sim \text { Gau }\left(\mu, \sigma^{2}\right) ; \\
& \mu \sim \mathrm{U}(-\infty, \infty) ; \text { and } \\
& \sigma^{2} \sim \operatorname{InvGamma}(1,0.01) .
\end{aligned}
$$

After a "burn-in" period of 200,000 iterations, we collected a sample of 40,000,000 and used a thinning of 4,000 due to the increasing autocorrelation between samples in the reversible jump MCMC scheme. The resulting size of the sample was 10,000, as for the other models. The approach, dealing with prior distributions, diagnostics and the choice of the thinning parameter, has been described in detail by Sturtz (2002).

The data and the results of the employed models for ischaemic heart disease in men are visualized in Fig. 3. (for 1980), Fig. 4. (for 1988) and Fig. 5. (for 1997) using quintiles. Table 3 shows the correlations between estimated SMR*s and ASMs.

In 1980 the original spatial structures of SMR*s and ASMs were approximately the same (Figs. 3a and $3 \mathrm{~b}$ ). We recognised only a few differences, particularly in the south-west of the state. Note that quintiles are drawn so that small discrepancies in the data set may result in a slightly different partition in the quintiles. Table 3 gives a detailed analysis of the number of quintile changes. The models produce slightly different results. The auto-Gaussian model

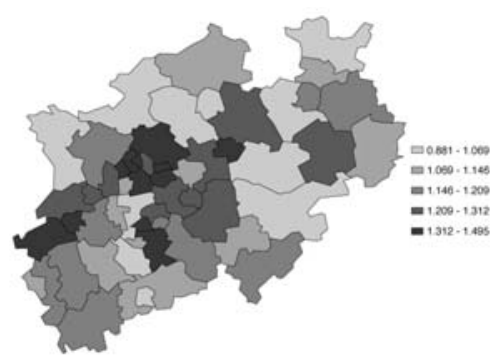

(a)

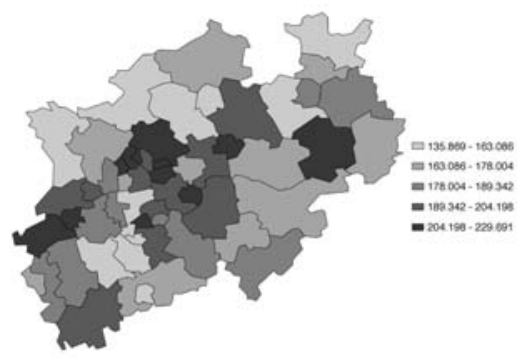

(b)

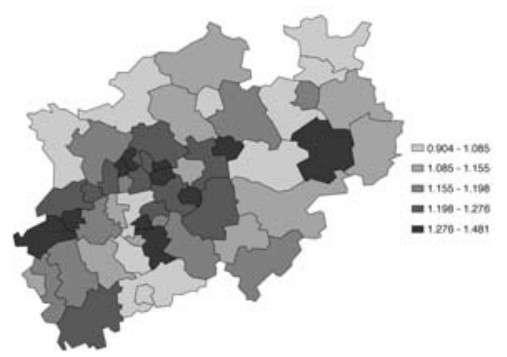

(c)

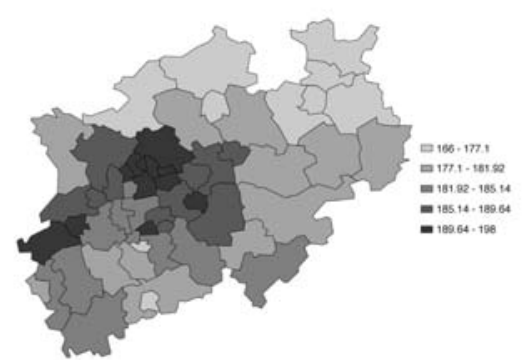

(d)

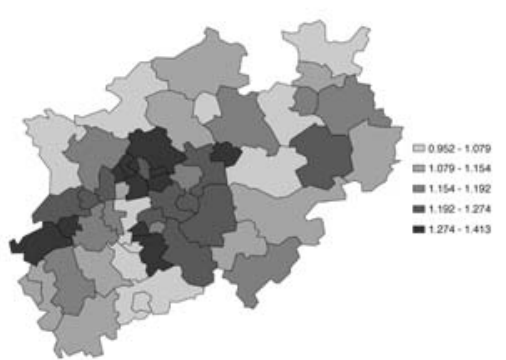

(e)

Fig. 3. Original spatial structure of SMR*s (a) and ASMs (b), smoothed SMR*s based on the Poisson-log-Normal model with spatial random effects (c), smoothed ASMs based on the auto-Gaussian model (d) and the application of the BDCD method on SMR*s (e) for ischaemic heart disease in men for North Rhine Westphalia, Germany, for the year 1980. 


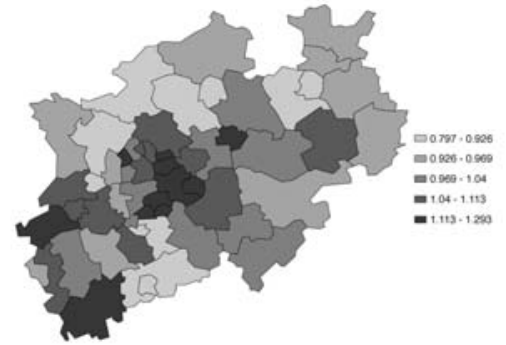

(a)

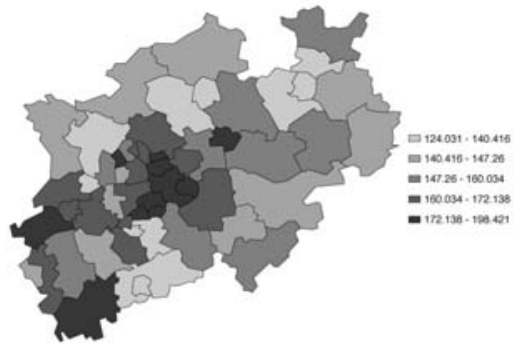

(b)

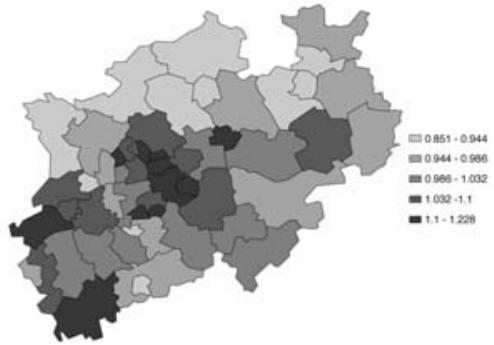

(c)

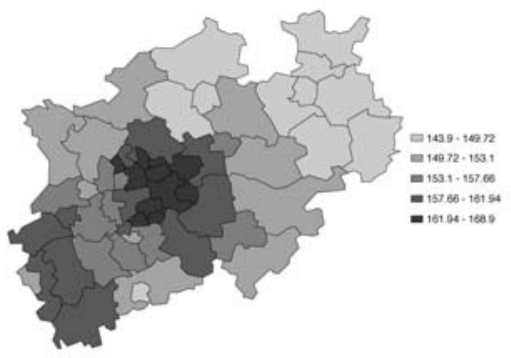

(d)

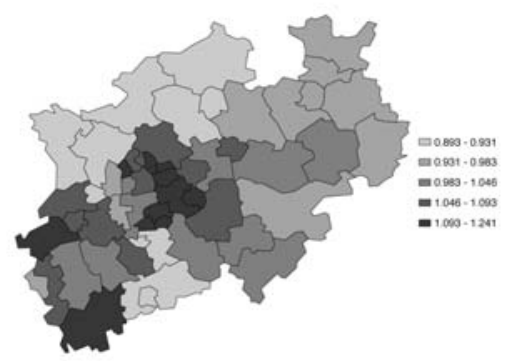

(e)

Fig. 4. Original spatial structure of SMR*s (a) and ASMs (b), smoothed SMR*s based on the Poisson-log-Normal model with spatial random effects (c), smoothed ASMs based on the auto-Gaussian model (d) and the application of the BDCD method on SMR"s (e) for ischaemic heart disease in men for North Rhine Westphalia, Germany, for the year 1988.

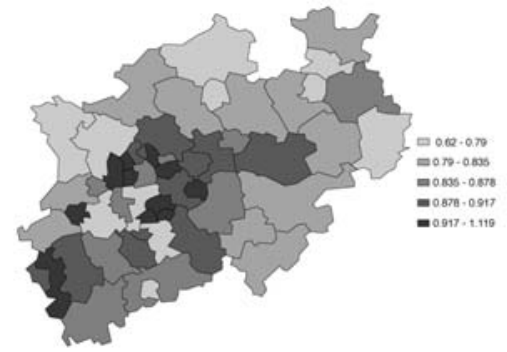

(a)

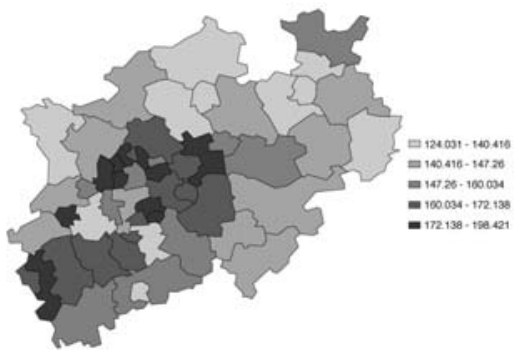

(b)

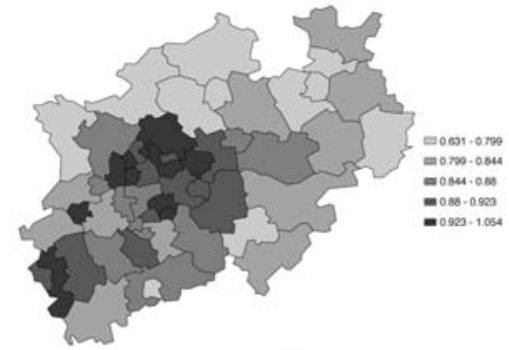

(c)

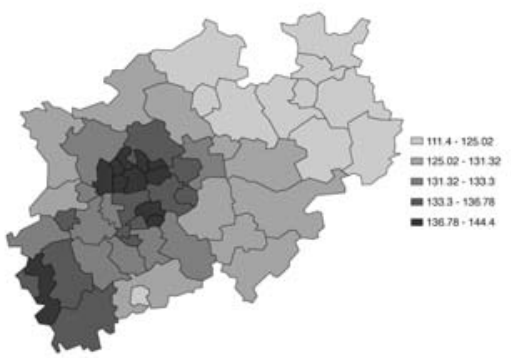

(d)

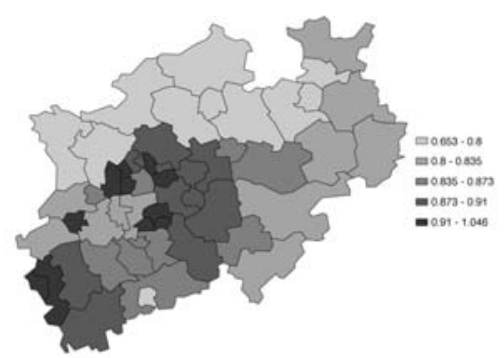

(e)

Fig. 5. Original spatial structure of SMR*s (a) and ASMs (b), smoothed SMR*s based on the Poisson-log-Normal model with spatial random effects (c), smoothed ASMs based on the auto-Gaussian model (d) and the application of the BDCD method on SMR*s (e) for ischaemic heart disease in men for North Rhine Westphalia, Germany, for the year 1997. 
gave the smoothest structure as depicted in Fig. $3 \mathrm{~d}$. With the other two models, the results were widely spread for the counties modelled with the $20 \%$ highest estimated death ratio, especially when employing the Poisson-log-Normal model with spatial random effects. Most differences were seen in the northern and the eastern part of the state but the estimated patterns were also similar to the original ones.

For the years 1988 and 1997, the spatial pattern of the age-standardised mortality ratio and the mortality rate were very similar (Figs. 4 and 5). In these two years, the differences between the three different models were negligible. Even if the number of differently allocated quintiles was high, the resulting pattern was similar since the association with low-risk and high-risk regions was similar by both approaches. For example, the highest death rates, both observed and estimated, were found to be in the middle of the state. We observed minor differences in quintile mapping, in particular in the less densely populated eastern parts of the area, both in 1988 and 1997.

There were higher differences in the modelled numbers. Again, we calculated the rank correlation coefficient to compare the models for the Poisson- distributed and for the Gaussian-distributed data. In this analysis, we included ischaemic heart disease in women as we observed a relation between the underlying population figures and the plotted quintiles when we analysed the raw data. The results are given in Table 3 .

The falling trend is still observable in estimated figures for both SMR*s and ASMs. Compared to the information in Table 1, the number of differently allocated counties with reference to male deaths increases. Nevertheless, despite being produced by different models, the correlation coefficient must still be deemed to be high. Furthermore, we obtained similar results when comparing the various possibilities of modelling SMR*s and ASMs. We conclude that differences in the presented values are not only due to the use of different models but also due to the arbitrary classification. Therefore, we recommend the inclusion of the true underlying figures instead of arbitrary classification structures obtained by percentiles for plotting risk surfaces. Nevertheless, due to the high correlation, any of the classifications may be employed.

Since our results were based on common causes-

Table 3. Overall SMR*s and ASMs and rank correlation coefficients for ischaemic heart disease. In the top (bottom) half SM̂R"s are calculated using the Poisson-log-Normal model with spatial random effects (the BDCD model), ASMs are calculated by the auto-Gaussian model.

\begin{tabular}{|c|c|c|c|c|c|}
\hline Sex & Year & SMR*s & ASMs & $\begin{array}{c}\text { Rank } \\
\text { correlation }\end{array}$ & $\begin{array}{l}\text { Counties } \\
\text { changing quintiles }\end{array}$ \\
\hline \multicolumn{6}{|c|}{ 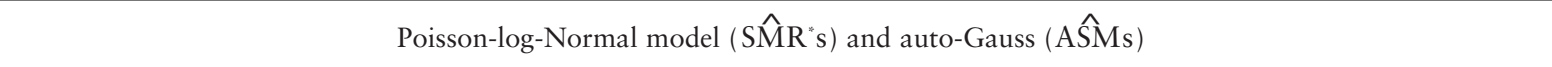 } \\
\hline Male & $\begin{array}{l}1980 \\
1988 \\
1997\end{array}$ & $\begin{array}{l}1.188 \\
1.020 \\
0.864\end{array}$ & $\begin{array}{l}183.10 \\
155.52 \\
131.10\end{array}$ & $\begin{array}{l}0.865 \\
0.905 \\
0.880\end{array}$ & $\begin{array}{l}21 \\
25 \\
26\end{array}$ \\
\hline Female & $\begin{array}{l}1980 \\
1988 \\
1997\end{array}$ & $\begin{array}{l}1.062 \\
1.021 \\
0.991\end{array}$ & $\begin{array}{r}72.23 \\
155.53 \\
62.22\end{array}$ & $\begin{array}{l}0.833 \\
0.745 \\
0.886\end{array}$ & $\begin{array}{l}26 \\
30 \\
21\end{array}$ \\
\hline \multicolumn{6}{|c|}{ BDCD ( $\widehat{\text { SMR"s }}$ ) and auto-Gauss (ÂMs) } \\
\hline Male & $\begin{array}{l}1980 \\
1988 \\
1997\end{array}$ & $\begin{array}{l}1.184 \\
1.019 \\
0.862\end{array}$ & $\begin{array}{l}183.10 \\
155.52 \\
131.10\end{array}$ & $\begin{array}{l}0.864 \\
0.878 \\
0.887\end{array}$ & $\begin{array}{l}22 \\
30 \\
26\end{array}$ \\
\hline Female & $\begin{array}{l}1980 \\
1988 \\
1997 \\
\end{array}$ & $\begin{array}{l}1.062 \\
1.021 \\
0.992 \\
\end{array}$ & $\begin{array}{r}72.23 \\
155.53 \\
62.22 \\
\end{array}$ & $\begin{array}{l}0.798 \\
0.710 \\
0.872 \\
\end{array}$ & $\begin{array}{l}28 \\
30 \\
21 \\
\end{array}$ \\
\hline
\end{tabular}


of-deaths, it was felt that it could be of interest to compare with outcomes in relation to rare causesof-death. Similar results were obtained when looking at the relation of both measures for rare causes-of-death from the same data base, e.g. AIDS (ICD 042-044).

\section{Discussion}

The SMRs* and the ASMs have been compared using mortality numbers for diseases with different risk pattern such as for ischaemic heart disease and lung cancer leading to a similar outcome in spatial as well as in temporal analyses. The rank correlation between both measures was high in all examples provided. Nevertheless, using quintiles changed the interpretation of plotted risk surfaces, as up to $50 \%$ of all regions were assigned to different quintiles. Since the rank correlation was high, differences in the models applied could not be the only reason for different quintile allocation. Hence, we recommend using a continuous colour scale for plotting the SMR*s and ASMs. The interpretation of such continuously shaded choropleth maps does not depend on any arbitrary classification used for interval shaded maps. Therefore, continuous shading is, in our opinion, preferable for all except spatial patterns with extreme outliers. Slocum et al. (2005) provides a good discussion on interval-shaded versus continuously shaded choropleth mapping as well as examples of both types from the geographic point of view.

In our example, the age-structure remained almost constant for all years investigated. Changing structures may change the resulting SMR*s as shown by Goldman and Brender (2000), but not necessarily the resulting ASMs due to direct standardisation. This type of standardisation ensures that adjusted observed rates reflect the age distribution of the chosen standard population, not the real population. However, to investigate the development of both measures when the age structure changes between different years of analysis, extensive simulation studies would be required. The inclusion of additive models in such a simulation study would allow to analyse robustness of SMR* versus ASMs, as discussed in Freeman and Holford (1980).

Alternative mortality measures might be used besides SMR *s and ASMs. For example, standardised rate ratios calculated by dividing the age-adjusted mortality rate for a study population by the age-adjusted mortality rate for a reference population. A simulation study based on the population of the United States comparing this type of standardisation to SMR*s has been carried out by Goldman and Brender (2000). They conclude that both measures lead to comparable results and interpretations, although the SMR*s are more affected by changes in the underlying age-structures.

\section{References}

Becker N, Frentzel-Beyme R, Wagner G, 1984. Atlas of cancer mortality in the Federal Republic of Germany. 2nd ed. Berlin, Springer.

Besag J, 1974. Spatial interaction and the statistical analysis of lattice systems, (with discussion). J R Stat Soc Ser B 43, 192-236.

Besag J, Kooperberg CL, 1995. On conditional and intrinsic autoregressions. Biometrika 82, 733-746.

Besag J, York J, Mollié A, 1991. Bayesian image restoration, with two applications in spatial statistics (with discussion). AISM 43, 1-59.

Best NG, Ickstadt K, Wolpert RL, 2000. Spatial Poisson regression for health and exposure data measured at disparate resolutions. J Am Stat Assoc 95, 1076-1088.

Best NG, Richardson S, Thomson A, 2005. A comparison of Bayesian models for disease mapping. Stat Methods Med Res 14, 35-39.

Breslow NE, Day NE, 1975. Indirect standardization and multiplicative models for rates, with reference to the age adjustment of cancer incidence and relative frequency data. J Chronic Dis 28, 289-303.

Clayton D, Kaldor J, 1987. Empirical Bayes estimates of agestandardised relative risks for use in disease mapping. Biometrics 43, 671-681. 
Cressie NAC, 1993. Statistics for spatial data. New York, Wiley.

DIMDI (editor), 1993. Internationale Klassifikation der Krankheiten (ICD). 9. Revision. $2^{\text {nd }}$ ed. Köln: Kohlhammer. Fernandez C, Green PJ, 2002. Modelling spatial correlated data via mixtures: a Bayesian approach. J R Stat Soc Ser B 64, 805-826.

Fleiss JL, Levin B, Paik MC, 2003. Statistics methods for rates and proportions. New York, Wiley.

Freeman DH, Holford TR, 1980. Summary rates. Biometrics 36, 195-205.

Gardner MJ, 1973. Using the environment to explain and predict mortality. J R Stat Soc Ser A 136, 421-440.

Goldman DA, Brender JD, 2000. Are standardised mortality ratios valid for public health data analysis? Stat Med 19, 1081-1088.

Green PJ, 1995. Reversible jump Markov chain Monte Carlo computation and Bayesian model determination. Biometrika 82, 711-732.

Green PJ, Richardson S, 2002. Hidden Markov models and disease mapping. J Am Stat Assoc 97, 1055-1070.

Knorr-Held L, Becker N, 2000. Bayesian modelling of spatial heterogeneity in disease maps with application to German cancer mortality data. All Stat Arch 84, 121-140.

Knorr-Held L, Besag J, 1998. Modelling risk from a disease in time and space. Stat Med 17, 2045-2060.

Knorr-Held L, Raßer G, 2000. Bayesian detection of clusters and discontinuities in disease maps. Biometrics 56, 13-21.

Mollié A, 1996. Bayesian mapping of disease, 1996. In: Gilks WR, Richardson S, Spiegelhalter DJ, editors. Markov chain Monte Carlo in practice. London: Chapman \& Hall, 359$380 \mathrm{pp}$.

Mollié A, Richardson S, 1991. Empirical Bayes estimates of cancer mortality rates using spatial models. Stat Med 10, 95-112.

Mungiole M, Pickle LW, Hansen-Simonson K, 1999. Application of a weighted head-banging algorithm to mortality data maps. Stat Med 18, 3201-3209.

Pickle LW, Mungiole M, Jones GK, White AA, 1999. Exploring spatial patterns of mortality: the new atlas of united states mortality. Stat Med 18, 3211-3220.

Pickle LW, White AA, 1995. Effects of the choice of age-adjustment method on maps of death rates. Stat Med 14, 615-627.

Pocock SJ, Cook DG, Shaper AG, 1982. Analysing geographic variation in cardiovascular mortality: methods and results (with discussion). J R Stat Soc Ser A 145, 313-341.

Rothman KJ, Greenland S, 1998. Modern epidemiology, $2^{\text {nd }}$ ed. Lippincott Williams \& Wilkins, Philadelphia.

Slocum TA, McMaster RB, Kessler FC, Howard HH, 2005. Thematic cartography and geographic visualization. $2^{\text {nd }}$ ed. Upper Saddle River, Pearson Prentice Hall.

Spearman C, 1904. The proof and measurement of association between two things. Am J Psychol 15, 72-101.

Spiegelhalter D, Thomas A, Best NG, Lunn D, 2004. WinBUGS users manual, version 1.4.1.

Sturtz S, 2002. Raum-zeitliche Untersuchung der HerzKreislauf-Mortalität in Nordrhein-Westfalen. Master's thesis. Department of Statistics, University of Dortmund.

Tsutakawa RK, Shoop GL, Marienfeld CJ, 1985. Empirical Bayes estimation of cancer mortality rates. Stat Med 4, 201-212.

Wakefield JC, Best NG, Waller L, 2000. Bayesian approaches to disease mapping. In: Elliot P, Wakefield JC, Best NG, Briggs DJ, editors. Spatial epidemiology: methods and applications. Oxford University Press, 104-127 pp.

Waller L, Gotway A, 2004. Applied spatial statistics for public health data. New York, Wiley. 